\title{
Vogl, Susanne
}

\section{Mit Kindern Interviews führen: Ein praxisorientierter Überblick}

Hedderich, Ingeborg [Hrsg.]; Reppin, Jeanne [Hrsg.]; Butschi, Corinne [Hrsg.]: Perspektiven auf Vielfalt in der frühen Kindheit. Mit Kindern Diversität erforschen. 2., durchgesehene Auflage. Bad Heilbrunn : Verlag Julius Klinkhardt 2021, S. 142-157

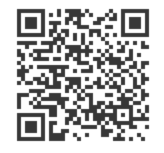

Quellenangabe/ Reference:

Vogl, Susanne: Mit Kindern Interviews führen: Ein praxisorientierter Überblick - In: Hedderich, Ingeborg [Hrsg.]; Reppin, Jeanne [Hrsg.]; Butschi, Corinne [Hrsg.]: Perspektiven auf Vielfalt in der frühen Kindheit. Mit Kindern Diversität erforschen. 2., durchgesehene Auflage. Bad Heilbrunn : Verlag Julius Klinkhardt 2021, S. 142-157 - URN: urn:nbn:de:0111-pedocs-222526 - DOI: 10.25656/01:22252

https://nbn-resolving.org/urn:nbn:de:0111-pedocs-222526

https://doi.org/10.25656/01:22252

in Kooperation mit / in cooperation with:

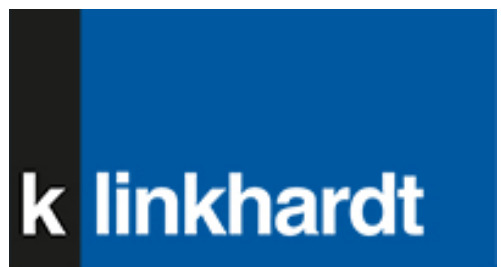

http://www.klinkhardt.de

\section{Nutzungsbedingungen}

Dieses Dokument steht unter folgender Creative Commons-Lizenz: http://creativecommons.org/licenses/by-nc-nd/4.0/deed.de - Sie dürfen das Werk bzw. den Inhalt unter folgenden Bedingungen vervielfältigen, verbreiten und öffentlich zugänglich machen: Sie müssen den Namen des Autors/Rechteinhabers in der von ihm festgelegten Weise nennen. Dieses Werk bzw. dieser Inhalt darf nicht für kommerzielle Zwecke verwendet werden und es darf nicht bearbeitet, abgewandelt oder in anderer Weise verändert werden.

Mit der Verwendung dieses Dokuments erkennen Sie die Nutzungsbedingungen an.

\section{Terms of use}

This document is published under following Creative Commons-License: http://creativecommons.org/licenses/by-nc-nd/4.0/deed.en - You may copy, distribute and transmit, adapt or exhibit the work in the public as long as you attribute the work in the manner specified by the author or licensor. You are not allowed to make commercial use of the work or its contents. You are not allowed to alter, transform, or change this work in any other way.

By using this particular document, you accept the above-stated conditions of use.

\section{Kontakt / Contact:}

\section{peDOCs}

DIPF | Leibniz-Institut für Bildungsforschung und Bildungsinformation Informationszentrum (IZ) Bildung

E-Mail: pedocs@dipf.de

Internet: www.pedocs.de

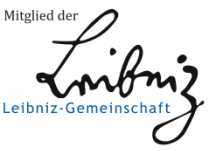




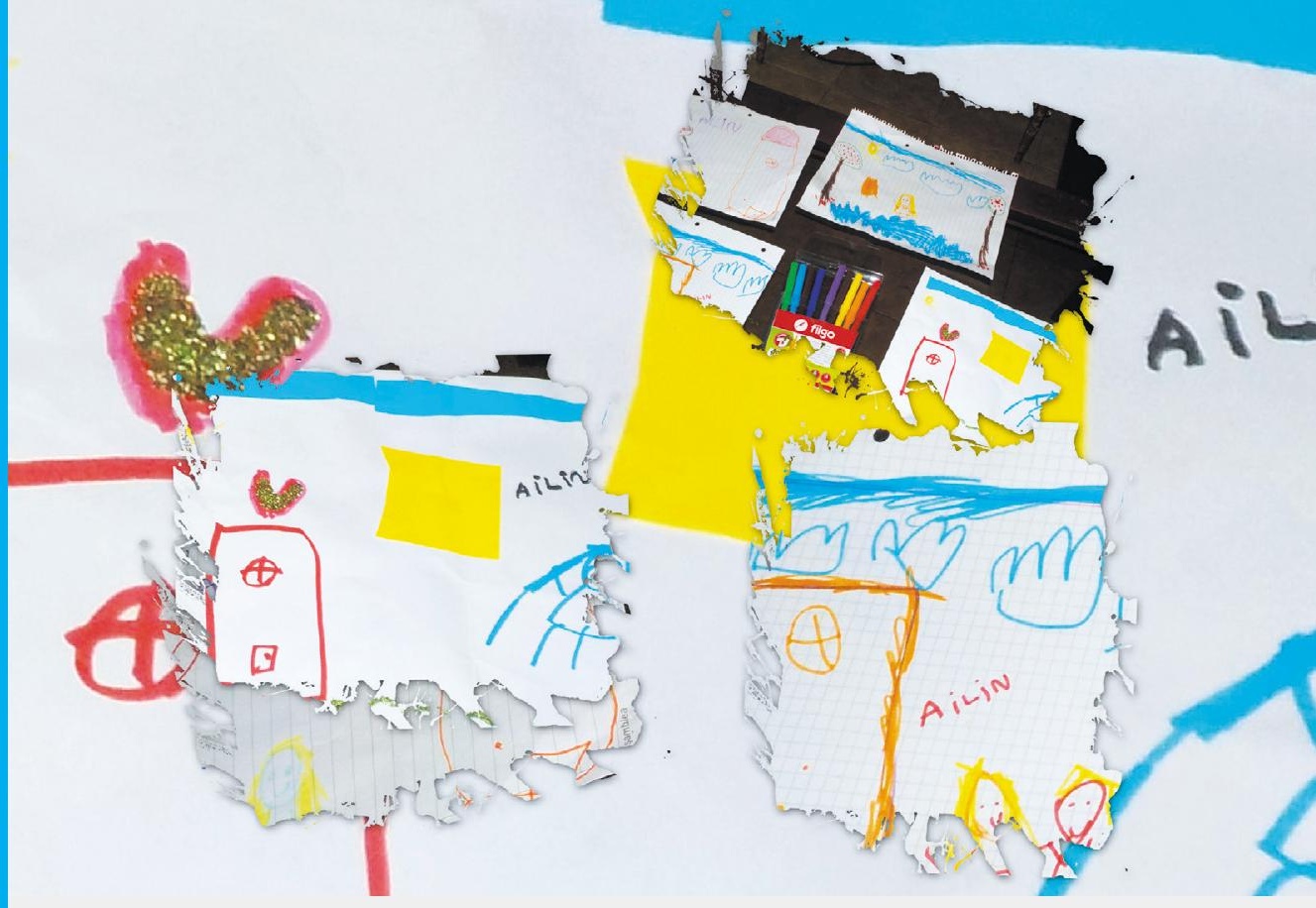

Ingeborg Hedderich

Jeanne Reppin

Corinne Butschi

(Hrsg.)

\section{Perspektiven auf Vielfalt in der frühen Kindheit}

Mit Kindern Diversität erforschen

2. Auflage 


\section{Ingeborg Hedderich Jeanne Reppin \\ Corinne Butschi \\ (Hrsg.)}

\section{Perspektiven auf Vielfalt in der frühen Kindheit}

Mit Kindern Diversität erforschen

2., durchgesehene Auflage 
Dieser Titel wurde in das Programm des Verlages mittels eines Peer-Review-Verfahrens aufgenommen. Für weitere Informationen siehe www.klinkhardt.de.

Bibliografische Information der Deutschen Nationalbibliothek Die Deutsche Nationalbibliothek verzeichnet diese Publikation in der Deutschen Nationalbibliografie; detaillierte bibliografische Daten sind im Internet abrufbar über http://dnb.d-nb.de.

2021.g. () by Julius Klinkhardt.

Foto Umschlagseite 1: Ailin, 5 Jahre, Provinz Entre-Rios, Argentinien und Corinne Butschi.

Druck und Bindung: Bookstation GmbH, Anzing.

Printed in Germany 2021.

Gedruckt auf chlorfrei gebleichtem alterungsbeständigem Papier.

(c) (1) $\Theta(D i e$ Publikation (mit Ausnahme aller Fotos, Grafiken und Abbildungen) ist veröffentlicht unter der Creative Commons-Lizenz: CC BY-NC-ND 4.0 International https://creativecommons.org/licenses/by-nc-nd/4.0/ 


\section{Inhalt}

Perspektiven auf Vielfalt in der frühen Kindheit.

Mit Kindern Diversität erforschen

Einleitung

Ingeborg Hedderich, Jeanne Reppin und Corinne Butschi

\section{Theorie}

Kindheit und Kindheitsforschung im Wandel

Corinne Butschi und Ingeborg Hedderich.

Erziehungswissenschaftliche Perspektiven auf Vielfalt, Heterogenität,

Diversity/Diversität, Intersektionalität

Katharina Walgenbach.

Vorderbühne - Hinterbühne. Zur Interdependenz der Horizonte von Diversität und Gleichheit

Cornelie Dietrich. 60

Differenzen und die Heterogenität von Kindern -

Einsätze blicktheoretischer Forschung

Friederike Schmidt.

\section{Methodologie und Methoden}

Kindgerecht forschen. Ein Überblick

Corinne Butschi und Ingeborg Hedderich.

Ankerpunkte, Wegmarken und Herausforderungen einer ethischen Forschung mit Kindern

Jeanne Reppin

Mit Kindern Interviews führen: Ein praxisorientierter Überblick

Susanne Vogl

Es ist noch jemand mit uns hier.

Puppet-Interviews in der Forschung mit Kindern

Marion Weise

Forschungsmethodische Vielfalt. Der Mosaic Approach

Sandra Schütz und Eva Theresa Böhm

Warum eigentlich? Philosophieren mit jungen Kindern

Martina Bernasconi. 


\section{Forschungszusammenhänge und Praxisreflexionen}

Ethnologische Kindheitsforschung im Überblick mit besonderer

Berücksichtigung der teilnehmenden Beobachtung

Werner M. Egli.....

Diversitätsbewusstes Denken und Handeln in Kindertageseinrichtungen -

Forschen mit Interviews und Interviewstreifzügen

Steffen Brockmann

Zur Relevanz der Zurechnung von Komplexität. Das Interview als

Methode der Datenerhebung im Alter früher Kindheit im Kontext von Behinderung

Martina Kaack.

Kamerakids: Forschen mit Photovoice

Corinne Butschi, Melike Hocaoglu, Manuel Zanardini, Valentin Mettler,

Ana Luisa Baumann-Santiago Martínez, Guillermina Chabrillon und

Ingeborg Hedderich.

Vielfalt in internationaler Zusammenarbeit erforschen. Ein Dialog

Corinne Butschi und Guillermina Chabrillon....

Einblick in ein vielfältiges Tätigkeitsfeld frühkindlicher Förderung in

Argentinien

Barbara Schoch

Das Erleben von Kindern mit Fluchterfahrung.

Forschen mit non-verbalen Zugängen

Marion Weise, Marion Lempp und Regine Morys

Wie erleben platzierte Vorschulkinder die Zugehörigkeit zu ihren

komplexen Beziehungswelten?

Forschen mit dem Geschichtenstammverfahren der

MacArthur Story Stem Battery

Maria Mögel.....

Lebenswelt Gemeinde: Mit Kindern forschen -

Aus Perspektiven und Methoden ein Mosaik zusammenfügen

Gabriela Muri und Heidi Simoni

Vom Schweizer Kindergarten ins Außerschulische, nach Ghana, und wieder zurück: Wenn Kinder und eine Ethnografin gemeinsam

,Grenzen' überschreiten

Ursina Jaeger

Die Autor*innen 


\section{Mit Kindern Interviews führen: Ein praxisorientierter Überblick}

\section{Einleitung}

Wenn man etwas von jemandem wissen will, fragt man. Das scheint naheliegend. Aber gilt das auch, wenn junge Kinder Gesprächspartner sind? Methoden der Sozialforschung - insbesondere Interviewformen - wurden für Erwachsene konzipiert (vgl. Kvale 2009; Vogl 2015a, 2015b). Doch kann man diese Methoden auch bei Kindern als Befragten einsetzen? Was sind methodische und praktische Besonderheiten in Interviews mit Kindern? Mit diesen Fragen beschäftigt sich der vorliegende Beitrag. Prinzipiell muss die Angemessenheit des Zugangs nicht nur im Hinblick auf das Erkenntnisinteresse, sondern auch auf die Zielgruppe geprüft werden. Zwar orientiert sich die Kindheitsforschung an Methoden der Sozialforschung, aber Forschungszugänge werden auch reflektiert und modifiziert (vgl. Heinzel, Kränzl-Nagl \& Mierendorff 2012). Da nur schwer allgemeingültige Regeln über die Anwendbarkeit verschiedener Methoden aufgestellt werden können, muss spezifisch für jede Fragestellung, für jede Methode und Technik entschieden werden. Ein Grundverständnis über altersspezifische Fähigkeiten und Entwicklungsherausforderungen helfen dabei. Daher werden in diesem Beitrag zunächst die Grundlagen der Interviewforschung und Anforderung an die Befragten erläutert, bevor auf sprachliche, kognitive und interaktive Fähigkeiten von Kindern eingegangen wird und praktische Tipps für die Interviewgestaltung gegeben werden.

\section{Grundlagen der Interviewforschung}

Interviews eignen sich gut, um das subjektive Verständnis von Personen zu untersuchen. Daher nehmen sie eine zentrale Stellung in der empirischen Sozialforschung ein und kommen häufig in der Kindheitsforschung zum Einsatz. Sie erscheinen als einfache und naheliegende Methode, um Informationen zu sammeln, auch aufgrund ihrer Nähe zu Alltagsgesprächen. Befragungen sind soziale Situationen, die auf wechselseitigen Erwartungen und Rollenzuschreibungen basieren. 
Die Interaktion ist in der Regel von (relativ) kurzer Dauer und sozial folgenlos, da es sich bei den Beteiligten um zwei (oder mehr) Fremde handelt.

Interviewformen können anhand unterschiedlicher Kriterien differenziert werden, z.B. dem Grad der Standardisierung - von vollständig standarisierten mit genau vorgegebenen Fragen und Antwortmöglichkeiten bis hin zu ganz offenen Befragungen, bei denen lediglich ein Erzählstimulus gegeben wird - oder den verwendeten Kommunikationskanälen - visuell und/oder auditiv, d.h. z.B. Telefon oder face-to-face Interviews. Je jünger die Kinder, desto angemessener ist in der Regel eine offene Herangehensweise mit möglichst vielen zur Verfügung stehenden Kommunikationskanälen. Offene Interviews bieten die Chance, sich tatsächlich dem Verständnis der Befragten und ihren Relevanzen zu nähern und ihnen nicht die eigenen Vorstellungen aufzuoktroyieren. Da Bedeutungsäquivalenz unter Kindern sowie zwischen Kindern und Erwachsenen nicht per se angenommen werden kann, braucht es gerade bei jüngeren Kindern mehr Raum, um flexibel auf die Fähigkeiten zu reagieren und in einen Aushandlungsprozess über Bedeutungen zu treten.

\section{Anforderungen an Befragte}

Befragte müssen in einem Interview parallel mehrere Aufgaben bewältigen, Sprachproduktion und Sprachverständnis sind nur ein Teil davon. Für funktionierende Kommunikation sind gleichzeitig linguistische sowie kognitive und soziale Fähigkeiten notwendig. Erinnerungsleistungen, Einschätzen der Anforderung der Kommunikationssituation, der Erwartungen der Interviewer*innen (und Forschenden), der Angemessenheit des eigenen Wissens im Hinblick auf diese Erwartungen, zu einem gewissen Grad die Steuerung der Kommunikation sind nur einige dieser gleichzeitig zu bearbeitenden Aufgaben. Diese Aufgaben können sich manchmal gegenseitig behindern, beispielsweise kann aus Unsicherheit oder wegen mangelnder sprachlicher Fähigkeiten das Erinnerte nicht angemessen wiedergegeben werden. Interviewsituation sind also sehr komplexe Interaktionssituationen. Dies kann gerade für jüngere Kinder leicht überfordernd sein.

\section{Fähigkeiten von Kindern}

In Interviews mit Kindern sind vor allem verbale, interaktive und kognitive Fähigkeiten entscheidend und determinieren die Erkenntnismöglichkeiten, die Anwendbarkeit und Ausgestaltungsmöglichkeiten von Befragungen. Ganz allgemein gilt, dass Fähigkeiten nicht plötzlich vorhanden sind, sondern sich entwickeln 
und zwar zunächst in vertrauten und wohl bekannten Themengebieten und erst später können sie auf neue Bereiche oder in neuen Kontexten angewendet werden (vgl. Garbarino \& Stott 1992). Der Schlüssel zum Erfolg von Kinderinterviews ist dementsprechend die Auswahl erfahrungsnaher Themen und bekannter Ausdrucksformen. Für die Planung von Befragungen muss also auch die Kontextabhängigkeit von Fähigkeiten berücksichtigt werden. Außerdem muss auf die Variabilität von Fähigkeiten innerhalb einer Altersstufe hingewiesen werden. Individuelle Unterschiede - auch in Bezug auf Charakter, Temperament, sozialen und ökonomischen Hintergrund - können teilweise in derselben Altersgruppe größer sein als altersübergreifende Unterschiede (vgl. Vogl 2012). Mit diesem Hinweis soll nun ein kurzer Überblick über die Entwicklung in den drei zentralen Fähigkeitsbereichen gegeben werden, um darauf aufbauend informierte Methodenentscheidungen treffen bzw. Methoden weiterentwickeln zu können.

\subsection{Sprachliche Fähigkeiten}

Die wichtigsten Voraussetzungen für Befragungen und gleichzeitig häufigste Einschränkung bei jungen Kindern sind natürlich sprachliche Fähigkeiten, die in linguistische und pragmatische Kompetenz unterschieden werden können - also das Wissen um Wortschatz und Struktur und die kontextangepasste Verständigungsfähigkeit (vgl. Grimm \& Weinert 2002). Ein kurzer Abriss über die Sprachentwicklung: Im Alter von zwei bis drei Jahren beginnen Kinder Erlebnisse in Form von Geschichten zu erzählen (vgl. Köhler 2001). Der Gebrauch von Sprache ist dabei sehr funktionalistisch und auf den Austausch von Informationen, Gedanken oder Konzepten beschränkt. Zwischen vier und fünf Jahren sind Kinder zunehmend daran interessiert, Informationen auszutauschen, Erfahrungen und Erlebnisse mitzuteilen, doch trotz größerem Wortschatz ist die Komplexität der ausgedrückten Ideen begrenzt. Erzählfähigkeit im linguistischen Sinn, bei der Erzählungen einem bestimmten Aufbau folgen (vgl. Schütze 1976), ist erst ab neun Jahren ausreichend ausgeprägt, um narrative Elemente aufzunehmen (vgl. Aldridge \& Wood 1998; Vogl 2015a).

Sprachproduktion und -verständnis stützen sich stark auf den konkreten Kontext. Um also Aussagen von Kindern zu verstehen, müssen Erwachsene auf ihre Beobachtungen und das Wissen über ein Kind in einer spezifischen Situation zurückgreifen. Während bei unter 7-Jährigen teilweise noch Wortschatzlücken auftreten, können Kinder mit sieben Jahren diese Lücken selbstständig durch Erklärungen und Umschreibungen überbrücken. Bis zu sieben Jahren verstehen Kinder Sprache noch sehr wörtlich, sind aber in ihrem Wortgebrauch weniger spezifisch (vgl. Holaday \& Turner-Henson 1989; Vogl 2012). Auch idiosynkratische Verwendung von Sprache findet sich bis zu diesem Alter (vgl. Garbarino \& Stott 1992). Gerade in der Kommunikation mit Fremden - wie in Interviews - ist kommunikative Kompetenz wichtig (vgl. Larsson 2005). Diese kommunikative Kompetenz 
bezeichnet die Fähigkeit, „Äußerungen unter Einbezug des situativen und sozialen Kontextes zu verstehen und zu produzieren" (Szagun 1996, 173). Entscheidend für gelingende Kommunikation ist die Fähigkeit zum Perspektivenwechsel, d.h. dass man sich in die Position eines anderen versetzen und so Erwartungen und Motive erkennen kann (vgl. Boueke \& Schülein 1991; Garbarino \& Stott 1992). Das fällt natürlich schwerer, wenn das Gegenüber unbekannt ist und nicht den gleichen Lebenskontext teilt, daher müssen Erwartungen klar expliziert werden (vgl. Cordón, Saetermoe \& Goodman 2005).

In einer Zusammenschau verschiedener Erkenntnisse zeigt sich: sprachliche Fähigkeiten sind kontext- und altersabhängig und variieren vor allem individuell. Geschlechterunterschiede nehmen tendenziell mit dem Alter zu, wobei Mädchen verbal versierter als Jungen sind (vgl. Fuhs 2000). Bereits mit fünf Jahren sind die sprachlichen Fähigkeiten so weit ausgeprägt, dass (qualitative) Interviews geführt werden können. Ab dann steht einer erfolgreichen Kommunikation nichts im Wege, wenn auch über das Gesagte noch wenig reflektiert wird (vgl. Trautmann 2010). Zu mangelnder Bedeutungsäquivalenz zwischen Befragten und Interviewer*innen kann es bei Kindern (und auch bei Erwachsenen) in jedem Alter kommen. Mit zunehmenden kommunikativen Fähigkeiten können Bedeutungen leichter und besser verhandelt werden.

\subsection{Kognitive Fähigkeiten}

Ein knapper Überblick über die Entwicklung kognitiver Fähigkeiten dient dazu, Leitfäden bzw. Fragen angemessen zu formulieren und zu strukturieren und Erkenntnismöglichkeiten realistisch einzuschätzen. Wichtige Aspekte sind das Gedächtnis, aber auch logisches und hypothetisches Denken.

Gedächtnisleistung und Erinnerungsstrategien nehmen während der frühen Kindheit stetig zu und sind ab ca. zehn Jahren vergleichbar mit Erwachsenen. Autobiografische Erinnerungen stellen ab etwa sieben Jahren kein Problem dar, es müssen aber geeignete Ausdrucksmöglichkeiten geschaffen werden. Längere selbstständige Erzählungen sind mit diesem Alter noch unrealistisch. Mit Gedächtnisstützen gelingen auch schon mit fünf Jahren mehr Erinnerungen (vgl. Greig, Taylor \& MacKay 2013), freier Recall ist ab sieben Jahren zunehmend möglich, wenn auch wenig Details gegeben werden (vgl. Poole \& Lamb 1998). Logisches und schlussfolgerndes Denken kann etwa ab sieben Jahren erwartet werden. In Ansätzen ist bei 5-Jährigen schon logisches und schlussfolgerndes Denken erkennbar (vgl. Mähler 2007). Besonders große Fortschritte sind diesbezüglich rund um das Schuleintrittsalter zu erwarten. Anfänge hypothetischen Denkens zeigen sich bereits ab sieben Jahren, ein deutlicher Entwicklungssprung findet zwischen neun und elf Jahren statt. Das heißt, abstrakte Probleme können erst vergleichsweise spät gelöst werden, hypothetische etwas früher. Begründungen werden $\mathrm{ab}$ dem Alter von neun Jahren dank Fortschritten im abstrakten und logischen 
Denken besser, relevanter und zielgerichteter. Das Denken wird differenzierter und es werden häufiger selbstständig Zusatzinformationen gegeben. Die Fähigkeit zu Verallgemeinerungen und einem argumentativen Aufbau von Begründungen zeigen sich erst später. Generalisierungen - wenn auch manchmal unangemessene - finden sich auch schon ab einem Alter von sieben Jahren. Ein Vermischen von Wunsch und Realität kommt bis zum Alter von fünf Jahren manchmal vor. Da aber ein solches Vermischen in aller Regel leicht zu erkennen ist, besteht dadurch nicht grundsätzlich eine Gefahr für die Datenqualität - geeignete Methodik vorausgesetzt.

\subsection{Interaktive Fähigkeiten}

Als zentrale Entwicklungsleistung für die Anwendbarkeit von Interviews kann die Fähigkeit zum Perspektivenwechsel angesehen werden (vgl. Vogl 2012). Die Fähigkeit zum Perspektivenwechsel ist wesentlich für die Interaktion mit anderen und entscheidend für das Gelingen von Kommunikationssituationen (vgl. Garz 2006). Nur wenn man sich anderer Perspektiven bewusst ist, können Handlungen und Gespräche koordiniert werden.

Die Fähigkeit zum Perspektivenwechsel und damit die Dezentrierung des Denkens entsteht zwar schon im Vorschulalter, für komplexe, neuartige Situationen ist sie erst mit etwa sieben Jahren verfügbar (vgl. Garbarino \& Stott 1992). Dadurch wird auch das Selbstkonzept stabiler und realistischer, wodurch sich Kinder besser beurteilen und im ,sozialen Raum' (vor allem bezogen auf Peers) verorten können (vgl. Berk 2010; Roebers 2007). Die Kooperations- und Kompromissfähigkeit steigt, genauso die emotionale Intelligenz. Ab ca. zehn Jahren können Kinder auch ,Generalisierte Andere' in Überlegungen miteinbeziehen.

Auch die Fähigkeit zum Perspektivenwechsel ist stark kontextabhängig (vgl. Garbarino \& Stott 1992). Alltagsnähe und Sinnhaftigkeit einer Frage aus den Augen der Kinder sowie die Verständlichkeit der Motive und Intentionen der handelnden Personen (vgl. Donaldson 1982) sind entscheidend. Werden also Ziele und Erkenntnisinteressen in Interviews klar kommuniziert sowie Fragen bzw. Aufgaben gestellt, die sich auf das Erfahrungswissen von Kindern beziehen, kann das Denken schon früher dezentriert werden. Reflexionen über sich selbst und andere sowie die Beziehung untereinander sind nicht unter sieben Jahren zu erwarten. Angemerkt sei, dass durch die neu gewonnen Fähigkeiten aber Nachteile für die Datenqualität entstehen können, weil mit besseren interaktiven und sozialen Kompetenzen auch die Fähigkeit zum Impression Management steigt bzw. überhaupt die ,Notwendigkeit ' von solchen Eindrucksmanipulationen erkannt wird (vgl. Vogl 2012). 


\subsection{Fähigkeiten sind nicht alles}

Kinder können am besten über ihre subjektiven Eindrücke, Bedürfnisse und Gedanken Auskunft geben. Allerdings ist es eine Sache, dass Kinder diese Dinge wissen, aber eine andere, dass sie sie in einer Befragung mitteilen (können). Der Schlüssel zum Erfolg von Kinderinterviews ist die Auswahl erfahrungsnaher Themen und bekannter Ausdrucksformen. Neben den Fähigkeiten und der Verfügbarkeit von Fähigkeiten in einem spezifischen Setting, ist es vor allem die Motivation, die Interviews scheitern oder erfolgreich sein lässt. So ist zum Beispiel neben der Erzählfähigkeit die Erzählfreudigkeit eine wesentliche Voraussetzung. Die Erzählfreudigkeit hängt stark von der Situation ab und erfordert ein gewisses Vertrauen unter den Beteiligten. Manche Kinder sind Fremden gegenüber sehr schüchtern und die Erzählfreudigkeit ist individuell sehr unterschiedlich. Diese Unterschiede sind weniger altersbedingt als durch Charakter, Temperament, sozialen und ökonomischen Hintergrund beeinflusst (vgl. Vogl 2012). In der Regel ist die Motivation von Kindern hoch (vgl. Vogl 2015a), wenn sie ehrliches Interesse spüren, nutzen sie gerne die Gelegenheit zu erzählen und genießen die Aufmerksamkeit (vgl. Lipski 1998).

\section{Herausforderungen bei Kindern als Befragten}

Einige Herausforderungen bei Kindern als Befragten können sein: das Autoritäts- und Generationenverhältnis, mangelnde Bedeutungsäquivalenz und Effekte der sozialen Erwünschtheit, aber auch die Interpretation gewonnener Ergebnisse. Die Fähigkeiten von Kindern werden häufig unterschätzt, weil ihre Fähigkeiten ,andersartig' sind. Womit man rechnen muss, sind eine kurze Aufmerksamkeitsspanne junger Kinder, ein wörtliches Sprachverständnis vs. unspezifischem Sprachgebrauch, Unsicherheit und Schüchternheit von Kindern, Probleme in der zeitlichen Verortung von Ereignissen (vgl. Poole \& Lamb 1998) und der Verwendung von Häufigkeits- und Mengenangaben. Zu erwarten sind Alterseffekte dahingehend, dass jüngere Kinder eher weniger sozial erwünschte Antworten geben und bei für sie relevanten und realitätsnahen Fragen auch wenig anfällig für Suggestion sind, ältere Kinder aber stärker daran interessiert sind, ein bestimmtes Bild von sich zu vermittelt (vgl. Vogl 2012). Jüngere zeigen dagegen etwas häufiger eine Ja-Sage-Tendenz, vermutlich auch dadurch begründet, dass nicht immer „weiß nicht“ geantwortet wird, wenn es eigentlich angebracht wäre. Die Erfassung sozio-demographischer Merkmale, v.a. der Herkunftsfamilie, ist nur sehr eingeschränkt möglich und sollte besser, ausgelagert' werden, indem hierzu z.B. ein kurzer Elternfragebogen erstellt wird. 
Ein Vermischen von Wunsch und Realität kommt bis zum Alter von fünf Jahren manchmal vor, ist aber in aller Regel leicht zu erkennen. Unter sieben Jahren sind Zeit- und Häufigkeitsangaben noch wenig reliabel, selbst die Worte „vorher" „nachher" können Probleme verursachen. Nach dem Schuleintritt wird das Zeitverständnis deutlich besser. Problematisch sind bei unter 10-Jährigen auch absolute Begriffe wie „immer“, „nie“ oder „alle“, genauso relative Angaben wie „oft“ oder „manchmal“ oder Zeitspannen wie „von morgens bis abends“ (vgl. Holaday \& Turner-Henson 1989; Vogl 2015a). Auch die Fähigkeit, Tage oder Zeiten zu identifizieren, ist bis zum Alter von zehn Jahren sehr begrenzt. Weil selbst ältere Kinder und Erwachsene sich nicht immer spontan und richtig erinnern, wann genau etwas passiert ist, ist es hilfreich, einen zeitlichen Rahmen mit einer weiter gefassten Frage zu umreißen.

Die Datenqualität ist insgesamt - wie auch bei Erwachsenen - personenabhängig. Hohe Motivation, Vertrauen und Selbstsicherheit sind der Datenqualität zuträglich. Zur Beurteilung der Datenqualität reichen aber Erwachsenenmaßstäbe nicht aus. Kinder sind nicht als ,kleine Erwachsene' zu verstehen. Sie erfahren und verstehen die Welt auf ihre eigene Weise. Ein/e erwachsene/r Forscher*in kann diese Perspektive nur bedingt einnehmen, aber diese Verschiedenartigkeit anzunehmen und zu akzeptieren ist der Ausgangspunkt.

\section{Mindestalter für Befragungen}

Ab welchem Alter Kinder sozialwissenschaftlich ,befragbar' sind, lässt sich nicht per se beantworten, sondern hängt vom Erkenntnisinteresse und den eingesetzten Methoden und Techniken ab. Als Empfehlung für qualitative oder wenig standardisierte Interviews gilt meist das Mindestalter von vier bis fünf Jahren (vgl. Bamler, Werner \& Wustmann 2010; Borgers, Leeuw \& Hox 2000; Heinzel 2003). Diese Empfehlung fußt vor allem auf Erkenntnissen zur Sprachentwicklung. In schriftlichen Befragungen sind aber beispielsweise entsprechende Lese- und Schreibkompetenzen notwendig, die frühestens mit acht Jahren vorausgesetzt werden können. Insgesamt stellen verschiedene Befragungsformen auch unterschiedliche Anforderungen an die Befragten, standardisiert mehr als offene. Deshalb hängt ein ,Mindestalter' stark von der Vorgehensweise und der Fragestellung ab.

Da Bedeutungsäquivalenz unter Kindern sowie zwischen Kindern und Erwachsenen nicht per se angenommen werden kann (vgl. Mey 2001), und „Zugang zur unbekannten Kultur der Kindheit" (Fuhs 2000, 88) geschaffen werden muss, braucht es gerade bei jüngeren Kindern mehr Raum, um flexibel auf die Fähigkeiten zu reagieren und in einen Aushandlungsprozess von Bedeutungen zu treten. Für standardisierte Befragungsmethoden wird daher ein Mindestalter von etwa zehn Jahren angenommen (letztlich abhängig vom Grad der Standardisierung und 
der inhaltlichen Ausgestaltung). Prinzipiell sind Kinder oft bessere Informanten als geglaubt, wenn man einen methodisch angemessenen Zugang zu ihrer Perspektive findet.

\section{Interviewgestaltung}

Nach diesem eher theoretischen Teil, wird nun das bisher Gesagte in konkrete Tipps für die Durchführung von Interviews. Der Fokus liegt auf allgemeinen Hinweisen zu Zugang, Ort, Zeit und technischen Hilfsmitteln, Gesprächsführung sowie Interviewtechniken.

\subsection{Zugang, Ort, Zeit und technische Hilfsmittel}

In der Regel ist man bei der Befragung von Kindern auf die Kooperation verschiedener Gatekeeper angewiesen. Nicht nur die Kinder selbst müssen in die Teilnahme einwilligen - das ist in der Regel erst der letzte Schritt, sondern vorgeschaltet ist noch die Bereitschaft der Direktor*in/Leiter*in, der Lehrer*innen/ Erzieher*innen und der Eltern. So gilt es bei der Rekrutierung der zu Befragenden mehrere ,Hindernisse‘ zu überwinden (vgl. Zartler 2018). Gatekeeper nehmen aber nicht nur auf die Auswahl der zu Befragenden einen Einfluss, sondern sie definieren auch die Situation mit.

Gleiches gilt für den räumlichen und zeitlichen Interviewkontext. Während zu Hause unter Umständen eine entspanntere Atmosphäre herrscht, können anwesende Dritte (z.B. Elternteil oder Geschwister) dem entgegenwirken. Die Schule dagegen ist mit Assoziationen wie Prüfung, Autoritätsverhältnis, Peergroup etc. verbunden, die ebenfalls das Verhalten der Kinder beeinflussen können. Mit dem Kontext verändert sich unter Umständen nicht nur die Auskunftsbereitschaft, sondern auch Bedeutungen können sich je nach Befragungssetting ändern (vgl. Scott 2000). „Bei der Auswahl des Raumes sollte seine Funktion nicht außer Acht gelassen werden: Kinder verhalten sich in einem ,Traumzimmer' anders als im ,Turnzimmer“" (Vogl 2012). Das gleiche Kind kann zuhause vorlaut und angeberisch sein, aber schüchtern und zurückhaltend in der Schule (vgl. Scott 2000). Allgemein gilt es, die Situation für die befragten Kinder so angenehm und entspannt wie möglich zu gestalten und eine Kommunikation ,auf Augenhöhe‘ zu ermöglichen. Insgesamt sollte der räumliche Kontext den Befragten möglichst vertraut sein und wenig Ablenkung bieten.

Wichtig ist auch, das Vertrauen der zu befragenden Kinder zu gewinnen. Dies kann nur gelingen, wenn das Interesse an deren Sichtweise und Lebenswelt glaubhaft vermittelt wird. Es darf nicht der Eindruck entstehen, dass die Kinder das letzte Glied in der Kette sind, das nach seiner Meinung gefragt wird, nachdem mit Lehrer*innen und Eltern gesprochen wurde. Das Vertrauen kann dadurch erhöht 
werden, dass die Kinder den/die Interviewer*in bereits vor der eigentlichen Befragung kennenlernen. Es hat sich bewährt, ein Vortreffen in einer Gruppensituation durchzuführen, bei der mehrere oder alle Teilnehm*innen an der Befragung ungezwungen informiert werden. Dieses Vorgehen hat den Vorteil, dass die Befragten sich im Schutze der Gruppe sicher fühlen und so Unsicherheit und Zurückhaltung abgebaut werden kann. Das Vortreffen soll einen Orientierungsrahmen bieten, in dem das Vorgehen und damit verbundene Erwartungen erläutert sowie der/die Interviewer*in und technische Hilfsmittel eingeführt werden.

Die Interviewdauer richtet sich auch nach der Aufmerksamkeitsspanne der Befragten. Erzählbereitschaft, Motivation, Interesse für das Thema und Ausgestaltung des Interviews haben einen Einfluss auf die maximal mögliche Länge eines Interviews. Es ist Aufgabe der Interviewer*innen, situativ auf Ermüdungserscheinungen der Befragten zu reagieren und gegebenenfalls das Interviews vorzeitig zu einem Abschluss zu bringen. Oft ist gerade die Dauer eines qualitativen Interviews vorab schwer einzuschätzen und variiert individuell sehr stark in Abhängigkeit von den Befragten.

Video- oder Ton-Aufzeichnungen sind in qualitativen Interviews meist unvermeidlich. Effekte der technischen Hilfsmittel sollten bedacht und minimiert werden. In der Regel werden die Geräte aber schnell uninteressant, vorausgesetzt die Kinder haben die Möglichkeit, ihre Neugier diesbezüglich zu stillen und gegebenenfalls die Geräte auch selbst auszuprobieren.

\subsection{Gesprächsführung}

Die Gesprächsführung mit Kindern folgt im Prinzip den gleichen Regeln wie bei Erwachsenen, allerdings sind manche Aspekte besonders zu berücksichtigen. Dazu gehört das Autoritätsgefälle und dass Kinder meist keine Vorerfahrung mit Interviews haben - Befragungssituationen werden eher mit Tests assoziiert. Daher bedarf es einer Erklärung der Gesprächssituation auf metakommunikativer Ebene und besonderer Anstrengungen, eine vertrauensvolle Atmosphäre zu schaffen. In der Gesprächsführung soll das Interesse an der kindlichen Perspektive und der Expertenstatus der Kinder verbal und nonverbal unterstrichen werden (Augenkontakt, aktives Zuhören). Als Gesprächsregel sollte auch erklärt werden, dass Schweigen und Nichtwissen akzeptabel sind.

Gesprächsführung im Interview bedeutet „Verkehr in beide Richtungen“ (Delfos 2004, 68). Besteht zwischen den Gesprächsteilnehmer*innen ein Machtgefälle, wie in Befragungen von Kindern, verfällt man im Gespräch leicht in einen ,Einbahnverkehr'. Daher ist es besonders wichtig, das Gespräch offen zu führen, damit die Themen und Äußerungen nicht eingeschränkt sind. Der/die Interviewer*in muss zwischen Beteiligen und Abwarten vermitteln. Von Interviewer*innen - gerade in qualitativen Interviews und insbesondere mit Kindern - wird Empathie verlangt, das heißt auch „Respekt vor der Eigenart der Kinder zu haben und als Erwachse- 
ner eine andauernde Haltung der Verwunderung einzunehmen statt einer - ungerechtfertigten - Allwissenheit über das, was in einem Kind vor sich geht" (Delfos 2004, 69).

Als Erwachsener neigt man sehr leicht dazu, voreilig Aussagen von Kindern zu interpretieren, etwas 'hineinzuinterpretieren' und Aussagen unterstützend zu ergänzen. Selbstbewusste Teilnehmer*innen widersprechen zwar ungerechtfertigten Unterstellungen, weniger selbstbewusste stellen solche Sachverhalte eher indirekt richtig. Es kann aber nicht immer davon ausgegangen werden, dass dies der Fall ist. Insofern ist Vorsicht geboten, insbesondere bei schüchternen, zurückhaltenden Kindern: bei diesen besteht zum einen vermehrt die Gefahr dieses unterstützenden Ergänzens und zum andern auch, dass sie nicht widersprechen, wenn die ,Unterstellung' nicht stimmt. Wenn ein Kind keine Antwort weiß oder eine Aufgabe/Frage zu schwer ist, sollte dem Kind bei der 'Lösung' geholfen werden, um Anspannung zu vermeiden. Dem Kind muss aus der Situation ,herausgeholfen' werden, ohne ihm das Gefühl des Versagens zu geben - auch wenn die Antwort dann eventuell nur begrenzt verwertbar ist (vgl. Vogl 2012).

Die gewählte Sprache soll dem Alter der Kinder angepasst sein, aber nicht verniedlichend wirken (kein Babytalk!), denn das Kind soll sich akzeptiert, ernst genommen und geschätzt, nicht ausgehorcht oder bewertet fühlen. Bedeutungsäquivalenz ist zwischen Kindern und Erwachsenen nicht immer gegeben, sondern muss manchmal erst hergestellt werden. Zeitliche Bezüge sind soweit möglich zu vermeiden oder mit besonderen Erinnerungshilfen zu verbinden. Da Kinder Verständnisschwierigkeiten oder fehlendes Wissen nicht immer verbalisieren, können Ja-Nein- oder Entscheidungsfragen wenig reliable Antworten provozieren. Besser eignen sich offene Fragen, vor allem nach Ja-Nein- oder Entweder-oder-Fragen, um die Güte zu evaluieren und Hintergrundinformationen zu erhalten.

Reden und Spielen zu kombinieren kann bei jüngeren Kindern hilfreich sein: Kinder haben bessere Fähigkeiten, mehrere Aktivitäten zu kombinieren, als Erwachsene. „Ein junges Kind wird oft besser bei einem Gespräch mitwirken, wenn es Spielen und Reden kombinieren kann. Das ist mit Sicherheit bis etwa acht Jahre der Fall“" (Delfos 2004, 93). Es ist aber darauf zu achten, dass die Aktivität nicht wichtiger wird als das Gespräch. Zu vermeiden sind außerdem Spieltechniken mit vielen Regeln (wie z. B. Gesellschaftsspiele) oder Spiele mit großem Lustgewinn, wie solche mit Sand, Wasser etc., die von der eigentlichen Fragestellung ablenken können. Besser ist es, wenn die Kinder ihre eigenen Lieblingsspielsachen mitbringen, weil sie ihnen vertraut sind (vgl. Berna 1994).

\subsection{Interviewtechniken und Hilfsmittel}

Bei Kindern werden häufig Hilfsmittel, wie Bildkarten, Fotos oder Handpuppen eingesetzt (vgl. Krüger 2006). Prinzipiell kann dabei zwischen kommunikationsanregenden und -unterstützenden Medien unterschieden werden. Beide Formen 
folgen der Grundidee, den Austausch in Befragungen zu befördern und die Motivation der interviewten Kinder zu erhöhen. Der Verbalanteil wird reduziert, die Situation durch einen Spielcharakter aufgelockert und das Autoritätsgefälle tendenziell relativiert (vgl. Kubisch \& Lampert 2000).

Zur Kommunikationsunterstützung werden häufig Handpuppen oder andere Figuren verwendet. Fragen werden vermittelt über eine Puppe an das Kind gerichtet und nicht , direkt' vom Interviewer/von der Interviewerin. Das Kind kann entweder direkt antworten oder ebenfalls vermittelt über eine Puppe. Vor allem im Vorschulalter bietet sich der Einsatz von Handpuppen an, weil es sich um vertraute Spielobjekte handelt. Handpuppen können auch als Projektionsfläche und damit als Abstraktionshilfe benutzt werden, gerade jüngere Kinder nehmen dies als vertraute Hilfe wahr (vgl. Kepper 1996; Vogl 2009).

Probleme beim Einsatz figuraler Kommunikationsmedien bestehen zum einen darin, dass sie ablenken können und zum anderen eben oft Antworten nicht eindeutig interpretierbar sind. Es ist in der Regel nicht zu klären, in welchem Ausmaß und in welche Richtung die Antworten durch das Kommunikationsmedium verändert wurden. Die Antworten können mehr oder weniger deutlich vom ,Alltagsdenken' des Kindes entfernt sein und es bestehen vermutlich auch interindividuelle Unterschiede in den Auswirkungen durch die Einführung eines Kommunikationsmediums (vgl. Lohaus 1989). Gerade dieser Spielcharakter kann die Kinder anregen, Wunsch und Fantasie in die Antworten einfließen zu lassen (vgl. Wittmann 1988). Mit anderen Worten: Auch wenn diese Technik für Kinder alltagsnäher ist, werden nicht zwingend realitätsnähere Antworten erzielt. Zu beachten ist außerdem, dass unterschiedliche Figuren unterschiedliche Effekte nach sich ziehen. Außerdem kann es problematisch sein, wenn Perspektivenübernahme oder ein hohes Abstraktionsniveau gefordert wird (vgl. Lohaus 1989).

Eine kommunikationsanregende Funktion können beispielsweise visuelle Stimuli wie Bilder, Fotos oder Gegenstände haben. Durch den zusätzlichen Reiz wird der anvisierte Bereich aktualisiert und es wird wahrscheinlicher, dass das Kind versteht, was der/die Forscher*in meint, weil durch Bilder der Gegenstand viel konkreter wird als durch bloße verbale Präsentation (vgl. Scott 2000). Außerdem kann anschauliches Material stärker als eine unmittelbare Befragung zur Motivierung der Kinder beitragen (vgl. Lohaus 1989) und Bilder etc. können zusätzlich Erinnerungsstützen sein (vgl. Scott 2000).

Im Rahmen von Interviews können auch Zeichnungen erstellt oder mitgebracht werden. Zeichnen gilt als mögliche Ausdrucksform für Emotionen, Intuition und Persönlichkeit (vgl. Kepper 1996). Zeichnungen bieten den „Vorteil, dass die Kinder auf nonverbale Art und Weise Dinge zum Ausdruck bringen können, die sie verbal noch nicht auszudrücken vermögen" (Kubisch \& Lampert 2000, 70). Sie können Grundlage für einen diskursiven Interpretationsprozess sein und die subjektive Sichtweise kann mit zusätzlichen Erläuterungen des Kindes rekonstruiert 
werden. Der Entstehungsprozess und die Kontextualität der Zeichnungen sind aber zu berücksichtigen (vgl. Mey 2005). Da Kinder mit unterschiedlichen Fähigkeiten meist gern zeichnen und malen, gilt der Einsatz solcher Techniken als inklusive und partizipative Form der Datengewinnung (vgl. Mukherji \& Albon 2010), wenngleich sie für Kleinkinder noch nicht geeignet sind.

Ähnlich können auch Fotos als unterstützende Medien verwendet werden. Die Befragten oder der/die Forschende können Fotos sammeln oder machen (vgl. Fuhs 2003). Fotos sind aber genauso wie Zeichnungen keine Abbilder der Realität. „Das Foto als Erzählstimulus in der qualitativen Forschung legt durch seine gegenständliche, themengebundene Darstellung (noch stärker als eine verbale Eingangsfrage) eine Erinnerungsarbeit entlang konkreter Situationen und Gegenstände nahe, ist aber gleichzeitig aufgrund der Vieldeutigkeit eines jeden Bildes offen für eine Vielzahl von Projektionen“" (Fuhs 2003, 280).

Erzählgegenstände sind, ähnlich wie Fotos, räumlich und zeitlich eng mit dem Thema des Interviews verbunden und fungieren als kommunikationsanregende Stimuli. Im Kontext des Interviews dienen sie als Gedächtnisstütze und Erzählhilfe (vgl. Fuhs 2000). Ähnlich verhält es sich mit Bildergeschichten, Cartoons mit auszufüllenden Sprechblasen (vgl. Zartler 2018) oder Geschichtenergänzungen: auf ein Thema fokussiert können Kinder ihre eigene Erfahrung und Sichtweise einbringen.

Neben kommunikationsunterstützenden bzw. -anregenden Medien werden in der Kindheitsforschung auch häufig assoziative und projektive Techniken eingesetzt. Eine Schwierigkeit dieser Techniken liegt in der Validität der Antworten, weil ihr Realitätsgehalt unter Umständen geringer als bei direkten Interviews ist. Andererseits sind eben direkte Fragen nicht immer möglich, sodass im konkreten Fall abgewogen werden muss, wie am besten vorgegangen wird. Bei assoziativen Techniken können visuelle Hilfsmittel (ab)lenken und projektive Techniken können bei jüngeren Befragten aufgrund mangelnder Fähigkeit zum Perspektivenwechsel problematisch sein und sollten durch, Validierungsfragen ' ergänzt werden, in denen eruiert wird, ob eigene Einstellungen und Meinungen tatsächlich projiziert werden.

Je nach Erkenntnisinteresse und zu untersuchender Altersgruppe eignen sich unterschiedliche Interviewmethoden. Es zeigen sich folgende Tendenzen: Im Prinzip können Erzählungen, Assoziationen und Projektionen (formal) ab neun Jahren erfolgreich eingesetzt werden. Unter diesem Alter ist manchmal mit Einschränkungen in der Erzählfähigkeit, dem Perspektivenwechsel sowie abstraktem und logischem Denken zu rechnen. 


\section{Zusammenfassung}

Kinder sind eine besondere Zielgruppe, bei der es nicht nur entwicklungsbezogene Aspekte zu berücksichtigen gilt, sondern beispielsweise auch (fehlende) Erfahrung mit Befragungssituationen (in der Regel sind das dann Prüfungssituationen), die Stellung in der Hierarchie gesellschaftlicher Wissensbestände sowie das spezifische Generationen- und Autoritätsverhältnis zwischen erwachsenem/r Forscher*in und jugendlichem/r Interviewten. Fasst man Erkenntnisse zu den Fähigkeiten von Kindern im Altersverlauf und den Hinweisen zur Planung und Durchführung von Interviews mit Kindern zusammen, ergeben sich einige „Dos and don'ts" der Gestaltung von Interviews. Der in der folgenden Tabelle angebotene Überblick soll helfen, für den eigenen Forschungskontext und die Planung und Durchführung von Interviews mit Kindern begründete Schlussfolgerungen treffen zu können. Ein wichtiger Hinweis: die Altersgrenzen sind nicht so strikt, wie die Tabelle suggeriert: Es sei nochmal auf die Variabilität innerhalb einer Altersgruppe und die Abhängigkeit vom Thema und Erhebungskontext verwiesen. Dennoch gibt es bestimmte Faustregeln, die man für das Design einer eigenen Studie berücksichtigen kann.

Tab. 1: Gestaltungstipps für Interviews mit Kindern

\begin{tabular}{|c|c|c|c|}
\hline & \multicolumn{3}{|l|}{ Alter der Befragten } \\
\hline & 4 bis 6 Jahre & 6 bis 8 Jahre & 8 bis 10 Jahre \\
\hline \multirow{3}{*}{ 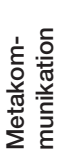 } & \multicolumn{2}{|c|}{ - Explizite Erklärung des Gesprächsrahmens } & - Gesprächsrahmen benennen \\
\hline & \multicolumn{3}{|c|}{ - viel Metakommunikation, um Erwartungen zu klären } \\
\hline & \multicolumn{3}{|c|}{$\begin{array}{l}\text { - Expertenstatus und Interesse an der kindlichen Lebenswelt verbal und nonverbal unter- } \\
\text { streichen }\end{array}$} \\
\hline \multirow{4}{*}{ 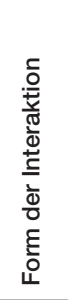 } & $\begin{array}{l}\text { - } \quad \text { spielen und reden } \\
\text { - } \text { zusätzlich nonverbale } \\
\quad \text { Spielformen }\end{array}$ & $\begin{array}{l}\text { - } \quad \text { reden und spielen } \\
\text { - } \quad \text { zusätzlich verbale } \\
\text { Spielformen }\end{array}$ & - reden, manchmal spielen \\
\hline & $\begin{array}{l}\text { - } 10 \text { bis } 15 \text { Minuten verbal } \\
\text { am Stück }\end{array}$ & $\begin{array}{l}\text { - } 15 \text { bis } 20 \text { Minuten } \\
\text { verbal am Stück }\end{array}$ & \multirow[t]{2}{*}{$\begin{array}{l}\text { - halbe bis Dreiviertelstunde verbal } \\
\text { am Stück }\end{array}$} \\
\hline & \multicolumn{2}{|c|}{ - stärkere Interviewer*innenbeteiligung erforderlich } & \\
\hline & \multicolumn{3}{|c|}{$\begin{array}{l}\text { - entspannte Atmosphäre schaffen (vor allem bei abstrakten und kognitiv anspruchsvollen } \\
\text { Fragestellungen) }\end{array}$} \\
\hline \multirow{4}{*}{ 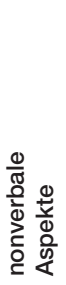 } & $\begin{array}{l}\text { - viel nonverbale Kommu- } \\
\text { nikation }\end{array}$ & \multicolumn{2}{|c|}{ - nonverbale Kommunikation } \\
\hline & 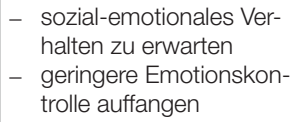 & & \\
\hline & \multicolumn{2}{|c|}{ - visuelle Reize lockern auf, können auch ablenken } & \\
\hline & \multicolumn{3}{|c|}{ - Interpretation visueller Reize abklären („was siehst du?“) } \\
\hline
\end{tabular}




\begin{tabular}{|c|c|c|c|}
\hline & \multicolumn{2}{|c|}{$\begin{array}{l}\text { - Fragen variiert wiederholen } \\
\text { - } \text { keine Zusammenfassung vom Kind erbeten }\end{array}$} & $\begin{array}{l}\text { - Zusammenfassen für die Struktur } \\
\text { und um sich über Verständnis zu } \\
\text { versichern }\end{array}$ \\
\hline & \multicolumn{2}{|c|}{$\begin{array}{l}\text { - Fragen nach Information } \\
\text { - Faktfragen } \\
\text { - Annähern durch Einzelfragen } \\
\text { - Vorschlagen von Antwortalternativen }\end{array}$} & $\begin{array}{l}\text { - Fragen nach Information, Meinun- } \\
\text { gen und Vorschlägen }\end{array}$ \\
\hline & \multicolumn{3}{|c|}{$\begin{array}{l}\text { - Recognition } \\
\text { - Fragen mit Bezug auf die Gegenwart }\end{array}$} \\
\hline & \multicolumn{3}{|c|}{ - Fragen nach Häufigkeitsangaben und zeitlichen Referenzen vermeiden } \\
\hline & - freien Recall vermeiden & - bedingt freier Recall & \multirow[t]{2}{*}{ - freier Recall } \\
\hline & \multicolumn{2}{|l|}{ - Erinnerungsstützen bieten } & \\
\hline & $\begin{array}{l}\text { - Warum-Fragen ver- } \\
\text { meiden }\end{array}$ & & $\begin{array}{l}\text { - Fragen nach Begründungen für } \\
\text { Meinungen etc. möglich }\end{array}$ \\
\hline & \multicolumn{2}{|c|}{ - Fragen nach Erklärungen vermeiden } & $\begin{array}{l}\text { - } \text { Fragen nach Erklärungen (vor allem } \\
\text { vertraute Abläufe) möglich }\end{array}$ \\
\hline & \multicolumn{2}{|c|}{$\begin{array}{l}\text { - Aufgaben mit logischem und schlussfolgerndem } \\
\text { Denken vermeiden }\end{array}$} & $\begin{array}{l}\text { - Aufgaben mit logischem Denken } \\
\text { und Schlussfolgern möglich }\end{array}$ \\
\hline & $\begin{array}{l}\text { - } \text { abstrakte und hypothe- } \\
\text { tische Fragen vermeiden }\end{array}$ & - abstrakte und hypot & etische Fragen möglich \\
\hline & $\begin{array}{l}\text { - } \text { keine Verallgemeine- } \\
\text { rungen fordern }\end{array}$ & - Verallgemeinerunger & möglich \\
\hline \multirow{2}{*}{ 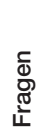 } & \multicolumn{2}{|c|}{$\begin{array}{l}\text { - Strukturierungsaufgaben in Erzählung abnehmen } \\
\text { - Erzählung durch Nachfragen aufrechterhalten } \\
\text { - Hintergrundinformationen erfragen }\end{array}$} & \multirow[t]{2}{*}{$\begin{array}{l}\text { - Projektion und hypothetisches } \\
\text { Denken möglich }\end{array}$} \\
\hline & \multicolumn{2}{|c|}{ - Projektionstechnik vermeiden } & \\
\hline
\end{tabular}

Quelle: Delfos 2004, 186 ; Vogl 2012, 314-315 ; Vogl 2015b

Welche Methoden für welche Kinder passen, das ist abhängig vom Alter, den Fähigkeiten, Vorlieben und Interessen. Genauso sind bestimme Methoden für bestimmte Fragestellungen besser geeignet als andere. Das Interview ist eine besondere, eben nicht alltägliche Kommunikationssituation. Anstatt nur die Fähigkeiten der Kinder infrage zu stellen müssen auch methodische Zugänge überdacht werden. Wenn wir wirklich kindgerechte Methoden entwickeln, können selbst junge Kinder gute Informanten sein. Anpassungsfähigkeit und Flexibilität in den Methoden und systematische, reflexive Methodenforschung ermöglichen einen angemessenen Zugang. Findet man einen geeigneten Zugang zu einem Kind, ist man immer wieder erstaunt über das Wissen und die Sichtweisen. 


\section{Literatur}

Aldridge, M. \& Wood, J. (1998): Interviewing children. Chichester.

Bamler, V., Werner, J. \& Wustmann, C. (2010): Lehrbuch Kindheitsforschung. Weinheim.

Berk, L. (2010): Development through the lifespan. Boston.

Berna, J. (1994): Die Verbalisierung in Erziehung und Kinderanalyse. In: G. Biermann (Hrsg.), Kinderpsychotherapie: Handbuch zu Theorie und Praxis (S. 75-94). Frankfurt a. M.

Borgers, N., Leeuw, E. de \& Hox, J. (2000): Children as Respondents in Survey Research. Bulletin De Méthodologie Sociologique, 66, 60-75.

Boueke, D. \& Schülein, F. (1991): Kindliches Erzählen als Realisierung eines narrativen Schemas. In H.-H. Ewers (Hrsg.), Kindliches Erzählen, Erzählen für Kinder. Weinheim/Basel, 13-41.

Cordón, I. M., Saetermoe, C. L. \& Goodman, G. S. (2005): Facilitating Children's Accurate Responses. Applied Cognitive Psychology, 19, 249-266.

Delfos, M. F. (2004): „Sag mir mal... “ Gesprächsführung mit Kindern (4-12 Jahre). Weinheim.

Donaldson, M. (1982): Wie Kinder denken. Bern.

Fuhs, B. (2000): Qualitative Interviews mit Kindern. In F. Heinzel (Hrsg.), Methoden der Kindheitsforschung. Weinheim, 87-103.

Fuhs, B. (2003): Fotografie und qualitative Forschung. In B. Friebertshäuser \& A. Prengel (Hrsg.), Handbuch Qualitative Forschungsmethoden in der Erziehungswissenschaft. Weinheim, 265-285.

Garbarino, J. \& Stott, F. M. (1992): What children can tell us. San Francisco.

Garz, D. (2006): Sozialpsychologische Entwicklungstheorien. Wiesbaden.

Greig, A., Taylor, J. \& MacKay, T. (2013): Doing research with children. London.

Grimm, H. \& Weinert, S. (2002): Sprachentwicklung. In: R. Oerter, L. Montada \& Oerter-Montada (Hrsg.), Entwicklungspsychologie. Weinheim, 517-536.

Heinzel, F. (2003): Qualitative Interviews mit Kindern. In B. Friebertshäuser \& A. Prengel (Hrsg.), Handbuch Qualitative Forschungsmethoden in der Erziehungswissenschaft. Weinheim, 396-413.

Heinzel, F., Kränzl-Nagl, R. \& Mierendorff, J. (2012): Sozialwissenschaftliche Kindheitsforschung. Theo-Web, 11, 9-37.

Holaday, B. \& Turner-Henson, A. (1989): Response effects in surveys with school-age children. Nursing Research, 38(4), 248-250.

Kepper, G. (1996): Qualitative Marktforschung. Wiesbaden.

Köhler, L. (2001): Zur Entstehung des autobiographischen Gedächtnisses. In: I. Behnken \& J. Zinnecker (Hrsg.), Kinder, Kindheit, Lebensgeschichte. Seelze-Velber, 65-83.

Krüger, H.-H. (2006): Forschungsmethoden in der Kindheitsforschung. Diskurs Kindheits- und Jugendforschung, 1(1), 91-115.

Kubisch, S. \& Lampert, C. (2000): Die Verwendung qualitativer Erhebungsmethoden in der Kinderfernsehforschung. TV Diskurs. (12), 68-71.

Kvale, S. (2009): Doing interviews. Los Angeles, Calif.

Larsson, A. S. (2005): Interviewing child witnesses. ISRN GU/PSYK/AVH: Vol. 152. Göteborg.

Lipski, J. (1998): Kindern eine Stimme geben. Zeitschrift für Soziologie der Erziehung und Sozialisation, 77-86.

Lohaus, A. (1989): Datenerhebung in Entwicklungspsychologie. Bern.

Mähler, C. (2007): Kindergarten- und Vorschulalter. In: M. Hasselhorn, W. Schneider \& J. Bengel (Hrsg.), Handbuch der Entwicklungspsychologie. Göttingen, 164-174.

Mey, G. (2001): Den Kindern eine Stimme geben! Aber können wir sie hören. Forum Qualitative Sozialforschung, 2(2), Art. 16, http://nbn-resolving.de/urn:nbn:de:0114-fqs0102160.

Mey, G. (2005): Forschung mit Kindern - Zur Telativität von kindangemessenen Methoden. In: G. Mey (Hrsg.), Handbuch Qualitative Entwicklungspsychologie. Köln, 151-183.

Mukherji, P. \& Albon, D. (2010): Research methods in early childhood. Los Angeles.

Poole, D. A. \& Lamb, M. E. (1998): Investigative interviews of children. Washington, DC. 
Roebers, C. (2007): Entwicklung des Selbstkonzeptes. In: M. Hasselhorn, W. Schneider \& J. Bengel (Hrsg.), Handbuch der Entwicklungspsychologie. Göttingen, 381-391.

Schütze, F. (1976): Zur soziologischen und linguistischen Analyse von Erzählungen. In: Internationales Jahrbuch für Wissens- und Religionssoziologie: Bd. 10. Beiträge zur Wissenssoziologie. Opladen, 7-41.

Scott, J. (2000): Children as Respondents. In: P. M. Christensen \& A. James (Hrsg.), Research with Children. London, 98-119.

Szagun, G. (1996): Sprachentwicklung beim Kind. Weinheim.

Trautmann, T. (2010): Interviews mit Kindern. Wiesbaden.

Vogl, S. (2009): Focus Groups with Children. In: J. Fiedler \& C. Posch (Hrsg.), Yes, they can! Children researching their lives. Baltmannsweiler, 86-98.

Vogl, S. (2012): Alter und Methode: Ein Vergleich telefonischer und persönlicher Leitfadeninterviews mit Kindern. Wiesbaden.

Vogl, S. (2015a): Children's Verbal, Interactive and Cognitive Skills and Implications for Interviews. Quality \& Quantity, 49(1), 319-338.

Vogl, S. (2015b): Interviews mit Kindern führen. Weinheim.

Wittmann, G. (1988): Über die Möglichkeit einer Befragung von Kindern. In: DJI (Hrsg.), Medien im Alltag von Kindern und Jugendlichen. München, 159-174.

Zartler, U. (2018). Kinder in der Familienforschung. In: O. Kapella, N. F. Schneider \& H. Rost (Hrsg.), Familie - Bildung - Migration. Opladen, 15-28. 